\title{
Variations saisonnières \\ de la densité apparente \\ et du taux d'infection \\ par Trypanosoma spp. \\ de G lossina palpalis gambiensis (Vanderplank, 1949) en zone soudanienne au Mali
}

\author{
A. Djiteye ${ }^{1}$ S.K. Moloo ${ }^{2}$ K. Foua Bi ${ }^{3}$ \\ E. Coulibaly ${ }^{1}$ M. Diarra ${ }^{1}$ I. Ouattara ${ }^{1}$ \\ D. Traoré ${ }^{1}$ Z. Coulibaly ${ }^{1}$ A. Diarra ${ }^{1}$
}

Mots-clés

Glossina palpalis gambiensis - Densité Variation saisonnière - Trypanosoma Infection - Végétation ripicole - Mali.

\section{INTRODUCTION}

La zone agropastorale de Baguinéda-Tienfala, située à l'est de Bamako, couvre de part et d'autre du fleuve Niger une superficie d'environ $1500 \mathrm{~km}^{2}$. Elle est comprise entre les parallèles

1. Laboratoire Central Vétérinaire, BP 2295, Bamako, Mali

2. ILRI, PO Box 30709, Nairobi, Kenya

3. FAST, Université d'Abidjan, 22 BP 582, Abidjan 22, Côte d'Ivoire

\section{Résumé}

Glossina palpalis gambiensis infeste les forêts ripicoles et les galeries forestières du fleuve $\mathrm{Niger}$ et ses affluents dans la zone agropastorale de Baguinéda-Tienfala. L'incidence de la trypanosomose (liée à la densité apparente de cette sous-espèce et à son infection trypanosomienne) variait en fonction de la saison et du gîte. En effet, au niveau du gîte de Tienfala (rive gauche du fleuve), la densité la plus élevée (21,70 glossines/piège/jour) a été observée en fin de saison des pluies, et la plus faible $(5,23)$ en saison sèche chaude. La sex-ratio était en général en faveur des femelles $(60,74$ p. 100). Au niveau du gîte de Baguinéda (rive droite), la densité la plus élevée $(8,70)$ a été trouvée en saison sèche froide, et la plus faible $(2,91)$ en fin de saison des pluies. La sex-ratio était en général en faveur des mâles (57,81 p. 100). L'infection trypanosomienne était plus élevée en fin qu'en début de saison des pluies et les taux observés variaient respectivement entre 6,66 et 10,68 p. 100 contre 0,48 et 1,48 p. 100 . Déterminées d'après leur localisation chez G. palpalis gambiensis, les infections étaient dues aux sous-genres Duttonella (Trypanosoma vivax : 80 p. 100), Nannomonas (T. congolense : 4 p. 100), Megatrypanum (T. grayi : 2 p. 100) et à des stades immatures localisés dans l'intestin moyen uniquement (14 p. 100). $12^{\circ} 24^{\prime}-12^{\circ} 48^{\prime} \mathrm{N}$ et les méridiens $7^{\circ} 30^{\prime}-8^{\circ} \mathrm{O}$. Le climat est de type soudanien, avec une pluviométrie annuelle moyenne de 1037 $\mathrm{mm}$ et une température annuelle moyenne allant de 26 à $35^{\circ} \mathrm{C}$. Glossina palpalis gambiensis infeste le cordon ripicole, les îlots et les galeries forestières du fleuve Niger et ses affluents sur environ $416 \mathrm{~km}$. Ces bandes forestières sont dominées par Syzygium guineense, Cynometra vogelii, Pterocarpus santalinoides. La savane arbustive de type soudanien est caractérisée par Vitelaria paradoxa, Parkia biglobosa, Terminalia spp. et Combretum spp. La savane herbeuse occupe le lit majeur du fleuve, avec une production fourragère moyenne de 2500 à $3000 \mathrm{~kg}$ de matière sèche par hectare. La zone d'étude comporte les forêts classées de Tienfala et de 
la Faya et le domaine rizicole de Baguinéda, avec 22300 ha et plus de $44 \mathrm{~km}$ de canaux d'irrigation. A l'intérieur de la « ceinture laitière » autour de la capitale, le cheptel bovin de cette zone est estimé à près de 40000 têtes. Il est composé essentiellement de races sensibles à la trypanosomose, zébus, animaux importés (Montbéliards), et de métis. L'élevage de telles races, avec la présence de la mouche tsé-tsé, comporte une contrainte majeure qui se résout par l'usage fréquent (mais souvent irrationnel) de produits trypanocides. La lutte contre la trypanosomose dans ce périmètre est une contribution aux efforts déployés pour améliorer l'alimentation et la situation économique des communautés rurales et urbaines de la ville de Bamako.

Avant toute action de lutte, il est nécessaire de connaitre le risque de la trypanosomose qui est fonction de certains facteurs liés au vecteur majeur, notamment sa densité et son taux d'infection. La présente étude porte précisément sur ces points.

\section{MATERIEL ET METHODES}

Deux gîtes de capture ont été choisis, distants d'environ $2 \mathrm{~km}$ et séparés par l'île de Senkoro :

- le gîte de Tienfala, à l'intérieur de la forêt classée qui porte son nom ;

- le gîte de Baguinéda, du côté du domaine agricole de Baguinéda avec plus de 22300 ha de terres irrigables.

Les enquêtes ont eu lieu aux périodes suivantes :

- saison des pluies (juillet-août) ;

- fin de saison des pluies (octobre-novembre) ;

- saison sèche froide (janvier-février) ;

- saison sèche chaude (avril-mai).

Cinq pièges biconiques Challier-Laveissière ont été posés tous les jours entre 6 et 19 h, à 100 m les uns des autres. Les glossines capturées ont été récoltées toutes les heures et mises dans des tubes à essai bouchés à l'aide de coton hydrophile.

La densité apparente (DAP) est le nombre moyen de glossines capturées par piège par jour.
La dissection en vue de la recherche des trypanosomes était faite sur place sous une loupe binoculaire. Après avoir dissocié les organes dans une goutte de liquide physiologique sur une lame porte-objet, les différentes préparations ont été recouvertes d'une lamelle et observées au microscope (objectif x 40) (4).

Le taux d'infection est le pourcentage de glossines infectées par des trypanosomes par rapport à la population observée.

\section{RESU LTATS ET DISCUSSION}

\section{Densités apparentes et sex-ratio de Glossina palpalis gambiensis}

Les résultats obtenus ont été consignés dans les tableaux I et II. D'une manière générale, la DAP était plus élevée au niveau du gîte de Tienfala $(13,88 \pm 2,06)$ que celui de Baguinéda $(4,76$ $\pm 1,02$ ). Cette différence était significative pendant toutes les saisons, sauf en saison sèche chaude où les DAP enregistrées étaient respectivement $5,23 \pm 1,73$ et $5 \pm 2,10$.

Sur la rive gauche (gîte de Tienfala), la DAP la plus élevée $(21,7$ $\pm 3,91$ ) a été observée en fin de saison des pluies, et la plus faible $(5,23 \pm 1,73)$ en saison sèche chaude. La sex-ratio était en faveur des femelles qui représentaient 60,74 p. 100 de l'échantillon total, mais l'inverse a été constaté en début de saison pluvieuse où le rapport mâles/femelles était égal à 1,31.

Sur la rive droite (gîte de Baguinéda), la plus forte densité $(8,70$ $\pm 4,44)$ a été atteinte en saison sèche froide et la plus basse $(2,91$ $\pm 1,04)$ en fin de saison pluvieuse. En général, les mâles $(57,81$ p. 100) étaient plus abondants que les femelles, sauf en fin de saison pluvieuse où l'inverse a été observé, mais la différence n'était pas significative au seuil de 5 p. 100.

\section{Infections de G. palpalis gambiensis par les trypanosomes}

Les mouches tsé-tsé adultes peuvent être infectées par les trypanosomes au cours des repas sanguins prélevés sur les hôtes vertébrés.

\section{Tableau I}

Variations saisonnières de la densité apparente et de la sex-ratio de Glossina palpalis gambiensis en zone soudanienne (fleuve Niger, gîte de Tienfala)

\begin{tabular}{|c|c|c|c|c|c|c|c|c|c|}
\hline \multirow[t]{2}{*}{ Saison } & \multicolumn{3}{|c|}{ Glossines capturées } & \multirow{2}{*}{\multicolumn{3}{|c|}{$\begin{array}{l}\text { Pourcentage de mâles } \\
\text { (Ecart réduit) }\end{array}$}} & \multirow{2}{*}{$\begin{array}{c}\text { Nombre jours } \\
x \text { pièges } \\
\text { par jour }\end{array}$} & \multirow{2}{*}{$\begin{array}{l}\text { Densité } \\
\text { apparente }\end{array}$} & \multirow[t]{2}{*}{ Variance } \\
\hline & Mâles & Femelle & Total & & & & & & \\
\hline Des pluies & 142 & 108 & 250 & $56,80 \%$ & $(3,04)$ & $\mathrm{S}$. & 25 & $10,00 \pm 3,91$ & 56,74 \\
\hline Fin saison des pluies & 210 & 441 & 651 & $32,25 \%$ & $(12,80)$ & $\mathrm{S}$. & 30 & $21,70 \pm 3,91$ & 119,44 \\
\hline Sèche froide & 190 & 349 & 539 & $35,25 \%$ & $(9,35)$ & $\mathrm{S}$. & 30 & $17,96 \pm 4,27$ & 142,51 \\
\hline Sèche chaude & 85 & 72 & 157 & $54,14 \%$ & $(1,46)$ & N.S. & 30 & $5,23 \pm 1,73$ & 23,49 \\
\hline Total & 627 & 970 & 1597 & $39,26 \%$ & $(12,13)$ & $\mathrm{S}$. & 115 & $13,88 \pm 2,06$ & 128,02 \\
\hline
\end{tabular}

Analyse de variance :

Saison des pluies/fin de saison des pluies : $t=4,527 \mathrm{~S}$.

Saison des pluies/saison sèche froide $: t=2,886 \mathrm{~S}$.

Saison des pluies/saison sèche chaude $: t=2,837 \mathrm{~S}$.

S. $=$ test statistique significatif au seuil de $5 \%$
Fin de saison des pluies/saison sèche froide $: \mathrm{t}=1,265$ N.S.

Fin de saison des pluies/saison sèche chaude $: t=7,545 \mathrm{~S}$.

Saison sèche froide/saison sèche chaude $: \mathrm{t}=5,411 \mathrm{~S}$.

N.S. = test statistique non significatif au seuil de $5 \%$ 


\section{Tableau II}

Variations saisonnières de la densité apparente et de la sex-ratio de Glossina palpalis gambiensis en zone soudanienne (fleuve Niger, gîte de Baguinéda)

\begin{tabular}{|c|c|c|c|c|c|c|c|c|c|}
\hline \multirow[t]{2}{*}{ Saison } & \multicolumn{3}{|c|}{ G lossines capturées } & \multirow{2}{*}{\multicolumn{3}{|c|}{$\begin{array}{l}\text { Pourcentage de mâles } \\
\text { (Ecart réduit) }\end{array}$}} & \multirow{2}{*}{$\begin{array}{c}\text { Nombre jours } \\
x \text { pièges } \\
\text { par jour }\end{array}$} & \multirow{2}{*}{$\begin{array}{l}\text { Densité } \\
\text { apparente }\end{array}$} & \multirow[t]{2}{*}{ Variance } \\
\hline & Mâles & Femelle & Total & & & & & & \\
\hline Des pluies & 80 & 60 & 140 & $57,14 \%$ & $(2,38)$ & $\mathrm{S}$. & 30 & $4,66 \pm 1,11$ & 9,74 \\
\hline Fin saison des pluies & 60 & 71 & 131 & $45,80 \%$ & $(1,35)$ & N.S. & 45 & $2,91 \pm 1,04$ & 12,80 \\
\hline Sèche froide & 101 & 73 & 174 & $58,04 \%$ & $(2,99)$ & $\mathrm{S}$. & 20 & $8,70 \pm 4,44$ & 102,83 \\
\hline Sèche chaude & 103 & 47 & 150 & $68,66 \%$ & $(6,46)$ & $\mathrm{S}$. & 30 & $5,00 \pm 2,10$ & 34,75 \\
\hline Total & 344 & 251 & 595 & $57,81 \%$ & $(5,38)$ & S. & 125 & $4,76 \pm 1,02$ & 34,46 \\
\hline
\end{tabular}

Analyse de variance :

Saison des pluies/fin de saison des pluies : $\mathrm{t}=2,181 \mathrm{~S}$.

Saison des pluies/saison sèche froide $: \mathrm{t}=2,050 \mathrm{~S}$

Fin de saison des pluies/saison sèche froide $: \mathrm{t}=3,408 \mathrm{~S}$.

Saison des pluies/saison sèche chaude $: t=0,279$ N.S

Fin de saison des pluies/saison sèche chaude $: t=1,911$ N.S

Baguinéda/Tienfala : $\mathrm{t}=7,921 \mathrm{~S}$.

Saison sèche froide/saison sèche chaude $: t=1,631$ N.S

S. = test statistique significatif au seuil de $5 \%$

N.S. = test statistique non significatif au seuil de $5 \%$

Les trypanosomes peuvent être transmis cycliquement par toutes les espèces de glossines en général ; mais l'importance d'une espèce en tant que vecteur dépend de ses habitudes alimentaires, de sa densité, et de sa répartition par rapport à celle des animaux domestiques.

Les préférences alimentaires des glossines influent sur les taux d'infection. Dans les régions où les glossines peuvent se nourrir sur des reptiles, le pourcentage de mouches infectées est très faible, par contre dans les localités où les animaux sensibles sont les sources de nutrition, on peut observer un pourcentage de mouches infectées aussi élevé que 38 p. 100 (1).

\section{Variations saisonnières du taux d'infection \\ de Glossina palpalis gambiensis}

Les taux d'infection les plus élevés (6,66 p. 100 et 10,68 p. 100) ont été enregistrés chez $G$. palpalis gambiensis en fin de saison des pluies, respectivement au niveau des gîtes de Baguinéda et Tienfala. Par contre, les taux les plus bas (1,48 p. 100 et 0,48 p. 100) ont été obtenus en début de saison des pluies (tableau III).

L'alternance des saisons en Afrique affecte l'épidémiologie des trypanosomoses du bétail, non seulement en raison des migrations imposées, mais aussi parce qu'elles influent sur la répartition et la densité des glossines. En saison sèche, les glossines se rassemblent dans les zones à micro-climat favorable à leur survie. Ainsi l'aire de dispersion des vecteurs se réduit, les possibilités de contact avec les hôtes mammifères diminuent. Toutefois les possibilités de contact sont accrues dans les régions où les glossines se concentrent près des abreuvoirs ou des pâturages de saison sèche (4).

Sur le ranch de Madina-Diassa (zone soudano-guinéenne, Mali), le taux d'infection de Glossina morsitans submorsitans variait d'une saison à l'autre ; par contre aucune différence significative n'a été trouvée chez G. tachinoides (3). L'incidence des trypanosomoses peut varier en fonction des saisons. En effet, d'après Challier (1), en Sierra Léone le taux d'infection du sous-genre Duttonella (type Trypanosoma vivax) est plus élevé pendant la période des fortes pluies que le reste de l'année. Le même fait a été observé chez $G$. palpalis, de juillet à septembre, au Nigeria du Nord.

\section{Variations du taux d'infection de G. palpalis gambiensis en fonction du sexe}

Dans la zone agropastorale de Baguinéda-Tienfala, les femelles de G. palpalis gambiensis $(5,95$ p. 100) ont été trouvées, de manière significative, plus infectées que les mâles (2,68 p. 100). Mais cette différence observée au gîte de Baguinéda (5,09 p. 100 contre 3,43 p. 100) n'était pas significative au seuil de 5 p. 100 (écart réduit $=0,81$ ) (tableau IV).

D'après Challier (1), de nombreux auteurs ont mentionné des infections plus importantes chez les mâles que chez les femelles, ou inversement chez les femelles que chez les mâles. Mais il faut de la prudence dans l'interprétation de ces différences, car il est possible que l'expérimentation provoque une mortalité différentielle des sexes et que l'échantillon ne soit pas représentatif.

En saison sèche chaude (avril, 1982) sur le ranch de MadinaDiassa, les femelles de G. morsitans submorsitans (21,1 p. 100) ont été trouvées plus infectées que les mâles (12,8 p. 100). Par contre, les captures effectuées sur véhicule (novembre, 1981) ont révélé un taux d'infection plus élevé chez les mâles (25,0 p. 100) que chez les femelles (12,5 p. 100) (3).

\section{Nature et localisation des infections}

D'après la localisation des 50 infections trypanosomiennes observées chez $G$. palpalis gambiensis :

- 40 (80 p. 100) se situaient uniquement au niveau de la trompe (sous-genre Duttonella : Trypanosoma vivax) ;

- 2 (4 p. 100) se trouvaient dans la trompe et dans l'intestin moyen (sous-genre Nannomonas : T. congolense);

- 1 (2 p. 100) était localisée au niveau de l'intestin postérieur (sous-genre Megatrypanum, T. grayi des reptiles) ;

- et 7 (14 p. 100) se trouvaient uniquement au niveau de l'intestin moyen. Elles étaient immatures ou bloquées, n'ayant pas pu achever leur développement en envahissant la trompe ( $T$. congolense), les glandes salivaires (T. brucei), ou l'intestin postérieur (T. grayi). 
Tableau III

Variations saisonnières du taux d'infection de Glossina palpalis gambiensis

$\begin{array}{lccccr}\text { Gîte } & \begin{array}{c}\text { Saison des } \\ \text { pluies }\end{array} & \begin{array}{c}\text { Fin de saison } \\ \text { des pluies }\end{array} & \begin{array}{c}\text { Saison sèche } \\ \text { froide }\end{array} & \begin{array}{c}\text { Saison sèche } \\ \text { chaude }\end{array} & \begin{array}{r}\text { Total } \\ \text { Tienfala }\end{array} \\ & 0,48 \% & 10,68 \% & 1,93 \% & 4,49 \% & (34 / 736) \\ \text { Baguinéda } & (1 / 206) & (25 / 234) & (4 / 207) & (4 / 89) & 4,10 \% \\ & 1,48 \% & 6,66 \% & 5,00 \% & 5,26 \% & (16 / 390) \\ \text { Total } & (2 / 135) & (4 / 60) & (5 / 100) & (5 / 95) & 4,89 \% \\ & & & 2,93 \% & (9 / 184) & (50 / 1126)\end{array}$

Ecart réduit :

Saison des pluies/fin de saison des pluies : e.r. $=5,16 \mathrm{~S}$.

Saison des pluies/saison sèche froide : e.r. $=1,94$ N.S

Saison des pluies/saison sèche chaude : e.r. $=2,95 \mathrm{~S}$.

S. = test statistique significatif au seuil de $5 \%$
Fin de saison des pluies/saison sèche froide : e.r. $=3,49 \mathrm{~S}$

Fin de saison des pluies/saison sèche chaude : e.r. $=1,95$ N.S.

Saison sèche froide/saison sèche chaude : e.r. $=1,11$ N.S.

\section{Tableau IV}

Variations du taux d'infection de Glossina palpalis gambiensis en fonction du sexe et du gîte

\begin{tabular}{|c|c|c|c|c|}
\hline Sexe & Tienfala & Baguinéda & Total & $\begin{array}{l}\text { Ecart } \\
\text { réduit }\end{array}$ \\
\hline Mâles & $\begin{array}{c}2,08 \% \\
(6 / 288)\end{array}$ & $\begin{array}{c}3,43 \% \\
(8 / 233)\end{array}$ & $\begin{array}{r}2,68 \% \\
(14 / 521)\end{array}$ & $\begin{array}{l}0,94 \\
\text { N.S. }\end{array}$ \\
\hline Femelles & $\begin{array}{r}6,25 \% \\
(28 / 448)\end{array}$ & $\begin{array}{c}5,09 \% \\
(8 / 157)\end{array}$ & $\begin{array}{r}5,95 \% \\
(36 / 605)\end{array}$ & $\begin{array}{l}0,52 \\
\text { N.S. }\end{array}$ \\
\hline Ecart réduit & $2,65 \mathrm{~S}$ & 0,81 N.S. & $2,65 \mathrm{~S}$ & - \\
\hline
\end{tabular}

S. = test statistique significatif au seuil de $5 \%$

N.S. = test statistique non significatif au seuil de $5 \%$

\section{CON CLUSION}

L'étude des populations de Glossina palpalis gambiensis révèle une différence dans l'abondance et la sex-ratio d'une rive à l'autre du fleuve Niger. La couverture végétale, plus dense sur la rive droite, joue un rôle très important en tant qu'habitat indispensable pour les espèces riveraines, mais n'est pas suffisante pour expliquer la différence des densités observées. A côté de la végétation, il y a lieu de tenir compte d'autres facteurs, tels que la présence ou l'absence de gîtes larvaires ou d'hôtes nourriciers pouvant provoquer des migrations. Compte tenu des conditions climatiques (hygrométrie et température), l'évolution de la densité des glossines semble normale au gîte de Tienfala.

En effet, la population de tsé-tsé croît après les premières pluies (conditions favorables) pour atteindre un optimum en fin de saison pluvieuse. Elle décroît légèrement en saison sèche froide en même temps que l'hygrométrie, pour atteindre son niveau le plus bas en saison sèche chaude quand les conditions climatiques sont les plus défa- vorables (hygrométrie faible et température élevée). La faible densité de G. palpalis gambiensis, observée au niveau du gîte de Baguinéda en fin de saison des pluies, pourrait s'expliquer par l'inondation des gîtes larvaires situés dans le lit majeur, la zone d'inondation.

Parmi les 50 infections observées chez G. palpalis gambiensis dans la zone d'étude, aucune infection du sous-genre Trypanozoon (T. brucei) n'a été notée. Néanmoins la maladie du sommeil existe, comme en témoigne l'infection en 1988 d'une fillette du village de Nyonya. Les résultats entomologiques obtenus concordent avec ceux des enquêtes menées sur les animaux par la Section protozoologie du Laboratoire central vétérinaire. Ils confirment que :

- le risque de la maladie est plus élevé en fin de saison des pluies ;

- le degré d'infection a été trouvé très faible pour les animaux des parcs situés loin des galeries forestières ;

- la quasi totalité des infections des bovins est due au sous-genre Duttonella (T. vivax).

En général, il apparaît que le sous-genre Duttonella (T. vivax) est plus apte à se développer chez les mouches tsé-tsé que les autres espèces de trypanosomes. Les études menées sur le ranch de Madina-Diassa (3) ont révélé que :

- la fréquence des infections du type T. vivax est plus élevée chez G. tachinoides (80,9 p. 100) que chez G. morsitans submorsitans $(59,4$ p. 100) ;

- les infections (probables) du sous-genre Nannomonas (T. congolense) sont moins fréquentes que celles du sous-genre Duttonella (T. vivax). Les fréquences observées chez G. morsitans submorsitans et G. tachinoides sont respectivement de 21,9 et 13,8 p. 100 ;

- les infections du sous-genre Megatrypanum (T. grayi) représentent 5,9 p. 100 chez G. morsitans submorsitans et 3,2 p. $100 \mathrm{chez}$ G. tachinoides ;

- les infections immatures observées représentent respectivement 20,6 et 4,3 p. 100 chez la sous-espèce de savane et chez l'espèce riveraine. 
Dans les conditions naturelles, on admet que la proportion de mouches tsé-tsé infectées par Trypanosoma brucei sensu lato est la moins élevée, soit au-dessous de 1 p. 100.

\section{Remerciements}

Nous tenons à remercier tous ceux qui nous ont permis de réaliser les enquêtes et d'achever ce travail, en particulier : Dr A.R. Gray, Dr M. Touré, Dr R. Eley (ILRI, Nairobi) ; Pr A. Aouti, Dr M. Dagnogo (FAST, RCI) ; Dr O. Diall, Directeur général du LCV ; Dr B. Seck et Dr C.F. Simbé, ancien DG et DGA du LCV ; Pr P. Ranque (OMS, Genève), Dr U. Feldmann (Joint FAO/IAEA Division, Vienne) ; Dr R. Cook, Directeur (Projet APEX/USAID, Bamako) ; Dr B. Kouyaté, Chef de la DDR du LCV ; et tous les autres collègues du Laboratoire central vétérinaire.

\section{BIBLIO GRAPHIE}

1. CHALLIER A., 1973. Ecologie de Glossina palpalis gambiensis, Vanderplank, 1949 (Diptera : M uscidae) en savane d'Afrique occidentale. Bondy, France, ORSTOM, 274 p. (Mémoire nº 64 )

\section{Summary}

Djiteye A., Moloo S.K., Foua Bi K., Coulibaly E., Diarra M., O uattara I., Traoré D., Coulibaly Z., Diarra A. Seasonal variations of the apparent density and trypanosome infection rates of Glossina palpalis gambiensis (Vanderplank, 1949) in the Sudanese zone of the Republic of Mali

G lossina palpalis gambiensis infests riparian and gallery forests of the Niger River and its tributaries in the agropastoral zone of Baguineda-Tienfala. The incidence of trypanosomosis in livestock (related to the apparent density of this tsetse subspecies and its trypanosome infection rates) varies depending on the season and the site. In Tienfala site, (left bank of the Niger River), the highest apparent density (21.70 tsetse/trap/day) was observed at the end of the rainy season and the lowest (5.23) during the hot dry season. The sex ratio was generally in favour of females $(60.74 \%)$. In the Baguineda site (right bank), the highest apparent density (8.70) was observed during the cold dry season, and the lowest (2.91) at the end of the rainy season. The sex ratio was generally in favour of males $(57.91 \%)$. The trypanosome infection rate was higher at the end than at the beginning of the rainy season and the observed rates varied between 6.66 and $10.68 \%$ against 0.48 and $1.48 \%$, respectively. Depending on the location in $\mathrm{G}$. palpalis gambiensis, the infections were due to subgenera Duttonella (Trypanosoma vivax : 80\%), $\mathrm{N}$ annomonas ( $\mathrm{T}$. congolense: $4 \%$ ), Megatrypanum (T. grayi : $2 \%)$ and to immature stages located only in the midgut (14\%).

Keys words: Glossina palpalis gambiensis - Density - Seasonal variation - Trypanosoma - Infection - Riparian vegetation Mali.
2. DAVID R., 1977. Study of a natural population of Glossina fuscipes fuscipes $\mathrm{N}$ ewstead and a model of fly movement. J. Anim. Ecol., 46: 309-330

3. DJITEYE A., 1985. Capacité vectorielle de Glossina (Diptera : Glossinidae) dans la transmission des trypanosomes en zone de savane soudano-Guinéenne (ranch de Madina-Diassa, cercle de Yanfolila). Thèse doct. de Spécialité, ISFRA, Bamako, Mali, 125 p.

4. ITARD J., 1981. Les trypanosomoses animales africaines. In : Précis de parasitologie vétérinaire tropicale. Maisons-Alfort, France, IEMVT, p. 398-428. (Manuels et précis d'élevage $n^{\circ} 10$ )

5. JACKSON C.H.N., 1933. On the true density of tsetse flies. J. Anim. Ecol., 2: 204-209.

6. PHELPS R.J., VALE G.A., 1978. Studies on populations of Glossina morsitans morsitans and $G$. pallidipes (Diptera: Glossinidae) in Rhodesia. J. Appl. Ecol., 15: 743-760.

7. SERVOZ H.M., 1987. Mission d'évaluation agropastorale et de formulations concernant le périmètre de Tienfala, République du Mali, mai 1987. Rome, Italie, FAO, GCP/RAF/191/ITA, 107 p.

Reçu le 3.4.96, accepté le 24.7.97

\section{Resumen}

Djiteye A., Moloo S.K., Foua Bi K., Coulibaly E., Diarra M., O uattara I., Traoré D., Coulibaly Z., Diarra A. Variaciones estacionales de la densidad aparente y de la tasa de infección por Trypanosoma spp. de Glossina palpalis gambiensis (Vanderplank, 1949) en la zona sudanesa en Malí

Glossina palpalis gambiensis infesta los bosques «ripícolas» y las galerías forestales del río Níger y sus afluentes en la zona agropastoril de Baguineda-Tienfala. La incidencia de la tripanosomiasis (ligada a la densidad aparente de esta subespecie y a su infección tripanosómica), varía en función de la estación y del sitio. En efecto, a nivel del sitio del Tienfala (márgen izquierda del río), la densidad más elevada $(21,70$ glosinas/trampa/día) se observó hacia el final de la estación lluviosa y la más baja $(5,23)$ durante la estación seca caliente. La relación de sexos fue en general favorable a las hembras $(60,74 \%)$. A nivel del sitio de Baguineda (márgen derecha), la densidad más elevada $(8,70)$ se presentó en la estación seca fría y la más baja $(2,91)$ hacia el final de la estación lluviosa. La relación de sexos fue en general favorable a los machos $(57,81 \%)$. La infección por tripanosomas fue más elevada al final que al principio de la estación de las lluvias y las tasas observadas varían, respectivamente, entre 6,66 y $10,68 \%$ contra 0,48 y $1,48 \%$. Determinadas de acuerdo a su localización en la G. palpalis gambiensis, las infecciones fueron debidas a los sub géneros Duttonella (Trypanosoma vivax: $80 \%$ ), Nannomonas (T. congolense: $4 \%$ ), M egatrypanum (T. grayi: $2 \%$ ) y a estadios inmaduros localizados unicamente en el intestino medio (14\%).

Palabras clave: Glossina palpalis gambiensis - Densidad Variación estacional - Trypanosoma - Infección - Vegetación ripícola - Malí. 